\title{
Paternités en exil: Une expérience thérapeutique père-enfant avec des réfugiés irakiens en Jordanie
}

\author{
Muriel Génot, Muath Asfoor et Hala Hammad
}

$\mathrm{D}$ epuis une quinzaine d'années, la prise en compte du rôle des pères dans le développement psychoaffectif de l'enfant commence à avoir droit de citée dans le cadre d'interventions périnatales au niveau microfamilial ${ }^{1}$. On sait par ailleurs que le fonctionnement de l'unité familiale est déstabilisé par l'expérience traumatique d'un ou de plusieurs de ses membres ${ }^{2}$, et se complique sous les effets du déracinement et de l'exil ${ }^{3}$. Néanmoins, lorsqu'il s'agit de s'attacher aux questions des conséquences transgénérationnelles du traumatisme, les études et pratiques se portent généralement vers les dyades mère-bébé4 ${ }^{\text {. Aussi le }}$ travail thérapeutique père-enfant constitue-t-il un terrain vierge pour la pratique humanitaire, particulièrement dans le cadre d'interventions psychosociales auprès de populations réfugiées 5 .

Rappelons que les expériences traumatiques - il serait plus juste de dire traumatogènes - ont pour principe d'action sur la psyché humaine de rendre le futur inaccessible, en ouvrant grandes les portes du passé sur le présent. Représentons-nous en effet que le traumatisme psychique est, comme son étymologie grecque le rappelle, «une blessure par effraction ». C'est l'événement qui a fait effraction. Alors que cet événement devient un événement du passé, une «menace interne» demeure installée dans la psyché sous la forme d'«une image traumatique ${ }^{6}$. Comme le décrit si clairement F. Lebigot, cette image "ne se comportera pas comme un souvenir: elle restera intacte au détail près et lorsqu'elle surgira à la conscience [...] ce sera toujours au temps présent, comme un événement en train de se produire» 7 . Le temps ne progresse plus et ne débouche alors plus sur rien. Ces conséquences individuelles d'une exposition traumatique, à fortiori lorsqu'il s'agit d'expositions multiples en temps de guerre et/ou résultant de la torture, nécessitent souvent des soins psychologiques ${ }^{8}$. L'évaluation de tels besoins parmi la population irakienne ayant trouvé refuge en Jordanie a justifié que l'organisation internationale Centre for Victims of Torture (CVT) ouvre un programme qui d'emblée accueille une grande proportion de clients $^{9}$ masculins. Si, depuis son ouverture, 78 pour cent des clients ont été suivis en thérapie de groupe (dont une moitié de groupes d'hommes), une proposition de soin individuel (alternative ou complémentaire au groupe) a été faite à 30 pour cent des clients. Les clients qui ont bénéficié $\mathrm{du}$ seul soin individuel sont en majorité des hommes; cette modalité d'intervention est généralement privilégiée dans les cas les plus sévères (le plus souvent des survivants de la torture). Au sein de l'équipe clinique, le constat a rapidement été établit que les interventions visant les conséquences individuelles doivent être accompagnées de dispositifs psychothérapeutiques complémentaires. C'est ainsi qu’ont été mis en place des dispositifs spécifiques accueillant des mères et leur bébé, des couples, et le dernier né des dispositifs, des pères et leur enfant.

Cet article rend compte de ce dernier type d'intervention. En l'absence de réflexions et d'expériences préexistantes pour travailler en groupe avec des couples père-enfant, le CVT n'a eu d'autre choix que d'inventer un dispositif psychosocial qui met l'écoute au centre de sa pratique.

Cette présentation analyse le processus thérapeutique sous un angle thématique, afin d'éclairer trois séries de problématiques qui ont émergé comme le produit de l'interaction entre les clients et l'équipe d'intervention: la maison, le refuge et le voyage; la fonction de père; la boîte de Pandore des questions (ou le sens donné aux événements du passé). Ces problématiques peuvent en particulier éclairer les débats en cours dans le champ des sciences sociales 
sur la question de «la capacité d'action» (agency) des réfugiés dans une situation temporaire qui perdure (ici dans le refuge urbain, ailleurs dans un camp, dans l'attente d'un retour ou d'une réinstallation dans un pays tiers). Notre approche thérapeutique montre que le temporaire n'est pas seulement une période où les réfugiés sont dans l'incertitude (dans les limbes) et dans l'incapacité d'être acteurs de leur propre devenir.

\section{Contexte et mise en place de l'intervention}

La population reçue par le CVT en Jordanie

Les données qui sont commentées ci-dessous ont été collectées depuis l'ouverture à la fin de 2008 d'un programme de soins psychologiques pour les survivants de la torture et les traumatisés de guerre. Les deux centres de $\operatorname{soin}^{10}$ du CVT ont accueilli à cette date 1200 clients irakiens. Parmi ces clients on compte 53 pour cent d'hommes pour 47 pour cent de femmes. Cette proportion est remarquable au regard des normes attendues lorsqu'il est coutume de penser qu'un homme, à fortiori originaire du monde arabe, est peu enclin à chercher le soutien d'un psychologue ${ }^{11} .34$ pour cent des clients traités ont traversé une expérience de torture ${ }^{12}$ (qu'elle soit ou non doublée d'une expérience traumatique liée à la guerre) dont deux tiers sont des hommes.

Les clients ${ }^{13}$ sont en majorité d'un niveau d'éducation supérieur, appartenant avant leur départ d'Irak à une classe sociale moyenne supérieure citadine. Nous reviendrons sur les conséquences de ces origines dans l'exil. Pour la plupart, ils vivent avec leur famille dans une grande ville en Jordanie. Or, 73 pour cent d'entre eux rapportent qu'ils n'ont plus suffisamment de moyens pour subvenir aux premières nécessités du foyer, notamment pour envoyer les enfants à l'école. Seuls 9 pour cent de nos clients ont actuellement un emploi; 3 clients sur 4 reçoivent une assistance humanitaire autre que celle du CVT. De l'autonomie financière en Irak, ils sont donc passés à la dépendance humanitaire en Jordanie.

Presque tous les Irakiens suivis dans nos centres sont enregistrés auprès du Haut Commissariat des Nations Unies pour les réfugiés (HCR) où ils ont généralement déposé une demande de réinstallation dans un pays tiers ${ }^{14}$. Ceux dont les demandes aboutissent seront vraisemblablement réinstallés aux États-Unis, le pays accueillant la majorité des demandeurs d'asile irakiens aujourd'hui. Une écrasante proportion de nos clients (81 pour cent) a quitté l'Irak à cause du conflit et vit l'exil depuis en moyenne trois ans. Leur récit fait généralement état de menaces reçues qui ont précipité le départ: menaces de mort ou d'enlèvement à l'encontre d'eux-mêmes ou de leurs proches (leurs enfants notamment) du fait de la politisation des identités tribales, confessionnelles et ethniques dans le conflit irakien ou du fait de leur proximité supposée ou réelle avec l'ancien régime de Saddam Hussein ou/et avec les forces de la coalition menée par les Américains. Les clients ayant subi la torture ont tous été détenus pour des durées variables après un enlèvement ou une arrestation arbitraire. Il n'est pas rare d'entendre que des rançons et autres dessous-de-table ont été payés, et que le commerce ou la maison familiale ont été spoliés ou détruits. On comprend que ces événements sont venus grever les économies et les ressources de la famille qui ne sont plus disponibles pour faire face aux nécessités de l'exil. Quelles que soient leurs expériences, les clients sont 83 pour cent à ne pouvoir s'imaginer retourner en Irak.

\section{Principes et naissance du dispositif père-enfant}

Ces éléments de contexte posés, il est temps de nous pencher plus avant sur leur impact psychosocial et en particulier lorsque l'on considère nos clients masculins. Le père dans la société irakienne est généralement reconnu comme une figure "d'autorité absolue», position qui s'assortit d'un nombre conséquent de devoirs ${ }^{15}$. Il est attendu des pères de famille en situation d'exil qu'ils remplissent le rôle de pourvoyeurs ${ }^{16}$ dans lequel ils se réalisaient pleinement en Irak (du fait de leur bonne situation socio-économique), et qui leur est socialement et culturellement dévolu. Cette attente émanant de l'entourage et de la société converge sur les pères qui eux-mêmes étaient accoutumés à se reconnaître dans ce rôle. De même, ils sont responsables d'assurer matériellement la continuité du développement des enfants (de l'apprentissage à la socialisation qui inclut la récréation et l'accès à certains biens), qui sont traditionnellement une ressource et une fierté ${ }^{17}$. De ces charges, dont les pères sont souvent en mal de s'acquitter, il ne reste plus que le poids d'une sorte de déchéance. Réfugiées en ville, les familles que nous recevons y trouvent une offre de consommation, de loisirs et de pratiques sociales au sein de laquelle elles savent rapidement s'orienter. Pour autant, faute de moyens, cette facilité leur demeure inaccessible, lorsqu'elle l'est pour d'autres Irakiens plus aisés et plus visibles dans l'espace urbain jordanien.

La perte de la sécurité matérielle ne trouve qu'une maigre compensation dans l'assistance. Cet aspect est particulièrement bien illustré dans l'étude récente ${ }^{18}$ réalisée auprès de 41 réfugiés irakiens réunis en groupes focaux par l'organisation AVSI: "Pour les participants, l'assistance humanitaire a pour objectif essentiel de restaurer la dignité et de permettre la construction d'un futur. Ainsi, quel que soit le montant de fonds qu'un programme reçoit ou les statistiques qu'il fournit, les organisations seront toujours évaluées par les participants à l'aune d'une comparaison entre ce qui a été gagné et ce qui a été perdu dans le passé». La pointe ultime de la fonction paternelle qui dresse un cadre protecteur en voyant plus loin dans le temps, en programmant 
le futur avant qu'il ne soit, se dissout dans l'incertitude de l'attente. La lenteur et le manque de transparence des procédures de demande d'asile, l'incertitude d'une réinstallation transforment le futur en rêve américain dont la réalisation semble toujours reportée à une échéance indéterminée.

À mesure que l'expérience permet au CVT d'affiner sa connaissance des besoins psychosociaux de sa clientèle, les indicateurs convergent pour justifier une proposition spécifique destinée aux pères. Indicateur extrême, des violences intrafamiliales dont les pères sont le plus souvent les acteurs nous sont parfois rapportées ${ }^{19}$. La violence domestique est un phénomène complexe et multifactoriel auquel le dispositif père-enfant ne se donnera pas pour objectif de répondre de manière spécifique. On peut néanmoins faire l'hypothèse que cette violence s'inscrit dans la longue mise en faillite d'une fonction paternelle à l'épreuve des traumatismes et de l'exil ${ }^{20}$.

La naissance du dispositif père-enfant du CVT trouve son origine dans les propos rapportés par J. au cours de ses premières séances de thérapie individuelle: «Depuis que je suis revenu de mon expérience de torture, je ne sais plus jouer avec ma fille». Ayant dit cela, J. se présente au centre pour la séance suivante avec sa petite fille de 6 ans. Le père et sa fille ne pouvant se résoudre à être séparés, cette dernière l'accompagne dans la salle de consultation (où elle n'a pas réellement sa place).

J. évoque spontanément un autre problème pour lequel il ne trouve pas de solution. Il a été enlevé par une milice alors qu'il était sorti pour une simple course domestique et maintenu en détention pendant un mois durant lequel il a été très sévèrement torturé; sa fille l'a vu disparaître brutalement puis réapparaître physiquement et psychologiquement brisé. Ni le père, ni sa fille - dit-il - ne sont dupes du roman familial ("papa était en voyage d'affaire») inventé pour faire face à l'indicible. Mais quelle est l'alternative?

Nous ${ }^{21}$ saisissant de ces deux niveaux de problématique, nous avons décidé de créer un dispositif qui permettait de les aider en parallèle. Avant de le décrire brièvement, nous présenterons en quelques mots les membres des groupes thérapeutiques impliqués. Chaque groupe est constitué de six membres, trois couples ou dyades père-enfant. Les six pères (dont J. fait partie) sont âgés de 25 à 50 ans et les six enfants (deux garçons et quatre filles ${ }^{22}$ ) ont entre 5 et 6 ans. Ce qui a présidé au choix des enfants, c'est leur âge: des enfants d'âge préscolaire qui ne peuvent encore compter sur un fonctionnement cognitif leur donnant plein accès à la rationalité au moment des séances ${ }^{23}$. Les enfants avaient entre 2 ans et 3 ans quand le conflit irakien s'est immiscé dans le cercle de leur intimité pour déstabiliser durablement l'équilibre familial et finalement provoquer l'exil (entre 2009 et 2010). Si quatre des pères parmi les six ont traversé un épisode spécifique de torture, qui s'est répété pour certains, les deux autres n'en rapportent pas, bien qu'ils aient été l'objet de menaces de mort en raison de leur affiliation familiale ou leur activité professionnelle. Qu'il s'agisse de menaces ou de torture, elles ont causé une séparation familiale plus ou moins longue (avant l'exil), les pères menacés ayant eu à s'éloigner pour tenter de préserver leur sécurité et celle de leur famille.

Tous les pères occupaient, avant que la situation de violence ne les en empêche, des emplois leur permettant de subvenir confortablement aux besoins de leur famille. Ils se retrouvent en situation de précarité financière en Jordanie et sont dans l'attente d'une réinstallation permanente dans un pays tiers.

$\mathrm{Au}$ moment où les séances commencent, les enfants ne fréquentent pas l'école. Aucun d'eux n'a été confronté à des expériences traumatiques repérées comme telles par les pères (même s'ils sont nés et ont grandi dans un contexte général de peur et d'insécurité qui s'est soldé par l'exil). Si ces dyades bénéficient de ce dispositif, c'est que les pères expriment eux-mêmes des difficultés dans leur interaction avec l'enfant, difficultés qui sont confirmées par l'entretien dyadique initial ${ }^{24}$.

\section{Objectifs, description et règles de fonctionnement $d u$ dispositif}

Le dispositif père-enfant est conçu comme un espace potentiel d'observation et d'expérience mutuelles. Il s'agit de promouvoir la capacité des pères à observer: l'enfant (le leur, un autre, plusieurs autres et pourquoi pas celui qu'ils portent en eux) et l'adulte (les autres pères, les thérapeutes) en interaction. La séance s'entend comme une parenthèse spatiale et temporelle au cours de laquelle il sera possible de se surprendre, d'être surpris et de prendre plaisir à être là (une invitation pour tous, adultes et enfants). Elle se présente aussi comme un espace de socialisation et de jeu pour les enfants. Une attention particulière est portée à l'agencement de la salle accueillant le groupe: un salon destiné aux pères ouvre, sans séparation visuelle, sur l'espace de jeu destiné aux enfants ${ }^{25}$.

Quelques règles, peu nombreuses mais essentielles, sont explicitées pour garantir la sécurité psychique de tous les membres du groupe (adultes et enfants): la séance s'inscrit dans un cadre temporel prévisible; nous nous réunissons à heure et jour fixes pour une durée d'une heure et demie chaque semaine et pour un nombre préétablis de séances (six au premier cycle, dix au deuxième $)^{26}$. La porte qui se referme en début de séance matérialise le fait que nous nous situons dans un espace où la confidentialité est de mise. Chacun y est libre de ses mouvements, de ses actions et de ses dires dans la mesure où ils ne nuisent pas à l'un des membres du 
groupe et qu'ils respectent l'intégrité des êtres et des lieux (on ne juge, ni ne crie, ni ne bat). Les objets contenus dans la pièce y demeurent pour la séance suivante et sont remis en ordre avant la clôture. Enfin, le dispositif n'est surtout pas une école pour les pères comme pour les enfants, mais il est pensé comme un temps de découverte et de nouveauté.

Lexplicitation détaillée des implicites théorico-cliniques du dispositif, bien qu'ils existent, n'est pas l'objet de cet article ${ }^{27}$. Il s'est agi d'une co-création dans la mesure où, sans que cela ne leur soit jamais demandé explicitement, les adultes comme les enfants ont joué un rôle décisif dans l'orientation du processus. Des interrogations ont émergé de la résonance dialectique entre nos hypothèses cliniques et la pratique; nous les avons élaborées en amont et en aval des séances.

\section{Problématiques saillantes apparues au cours $d u$ processus thérapeutique}

Le thème de la maison fut l'un des tous premiers à émerger lors du travail en groupe (première séance du premier cycle); la fonction de père s'est invitée dans un deuxième temps (au début du second cycle); la boîte à questions fut ouverte lorsque nous avons abordé le milieu du second cycle. Il est bien entendu que ces trois thèmes s'articulent les uns aux autres et les présenter séparément vise à faciliter leur analyse. Préciser leur ordre d'apparition au cours du processus permet néanmoins de mieux saisir les enjeux d'emboîtement auxquels se confrontent ces paternités en exil au-delà de trajectoires strictement individuelles.

La maison, le refuge et le voyage

Les réfugiés ont par définition quitté leur maison. Qu'est-ce que cela signifie pour eux?

Vignette 1: Lors de la toute première séance, la consigne est donnée à chacune des deux dyades présentes ${ }^{28}$ de créer ensemble quelque chose qui les représente. Les enfants prennent l'initiative et dessinent une scène similaire: un enfant qui joue à l'extérieur. Une façon pour eux de figurer la tâche de leur père: les regarder jouer. Il faut trouver un titre à chaque scène et un premier père nomme le dessin de sa fille "la maison du futur» (il précise "aux États-Unis»). Lorsque nous lui demandons où se trouve la maison qui n'est pas représentée, il la dessine lui-même. L'autre enfant, un garçon, a dessiné une scène où une voiture menace de renverser un enfant qui joue ${ }^{29}$. Lorsque la thérapeute pose la question: «Y a-t-il un endroit où on peut se sentir en sécurité ?" - le père et le fils dessinent ensemble une maison puis le fils demande au père de les représenter tous deux à l'intérieur. La scène est nommée de l'association de leurs deux prénoms.
De cette séquence, la maison se dégage comme référent commun aux pères et aux enfants. La maison est d'emblée manquante ou absente parce qu'elle a été perdue dans le passé et qu'elle existe au futur comme un rêve. Mais la maison est aussi garante de sécurité (au regard des dangers du dehors) et de continuité (si l'on peut s'imaginer y vivre plus tard, ailleurs), et ce sont aussi deux nécessités du présent. Comment réussir à reconstruire dans l'exil temporaire ces fonctions essentielles? C'est en prenant la mesure de ce besoin que la lecture d'un récit illustré, La Tempête ${ }^{30}$, a été proposée à nos groupes. Ce livre raconte l'histoire d'une enfant-souris dont la maison est emportée par la tempête. Son lit d'enfant-souris se transforme en tente-radeau de sauvetage, ballottée par les flots, au creux de laquelle viennent se réfugier le père et la mère souris. La famille se serre autour des quelques objets familiers que le père a pu sauver des eaux. L'histoire se termine sur une question de l'enfant à sa mère:

«Il demande:- Qu'est-ce qu'on fait maintenant?

— On voyage, dit Maman ${ }^{3}{ }^{31}$

Vignette 2: Pour la séance évoquée ici, il a été décidé de diviser le groupe en deux sous-groupes, les pères d'un côté, les enfants de l'autre, occupant chacun un espace de notre pièce habituelle. Avec le soutien d'un thérapeute dans chaque sous-groupe, ils sont invités à évoquer un moment de séparation d'avec l'autre. Parmi les pères (qui, dans ce premier cycle, ont tous été enlevés et torturés), un seul est vraiment en mesure de répondre à la consigne en évoquant, à mots couverts, cet évènement extrême. Un autre, comme pour en conjurer le danger existentiel, nie purement et simplement la possibilité d’une séparation: "Je ne peux pas me séparer [de mes enfants] sinon je perds le sens de ma vie. » Les deux autres pères s'engouffrent alors dans son sillage et font référence à la norme sociale: «Dans notre culture [...] un père doit être présent à la maison.» Du côté des enfants, ce sont les dessins qui parlent pour refuser la séparation: ils se sont représentés aux côtés de leur père; et sur chaque dessin à côté du couple père-enfant, il y a un avion ou un vaisseau volant.

L'évocation de la séparation ramène à l'indicible du passé, à ce qui n'aurait pas dû se passer et in fine à ce qui a été perdu. Dans un élan vital, les enfants se mettent en mouvement dans l'intention d'oublier ce passé et de transformer l'exil en voyage pour lequel juste un moyen de transport est nécessaire.

Vignette 3: Lors du deuxième cycle, nous introduisons une tente pour enfant dans la salle de consultation. Spontanément ceux-ci y trouvent refuge pour le rituel qui clôture chaque séance en musique. Se soustrayant quelques minutes au regard de leur père, les enfants élisent cette maison-tente pour faire le voyage de la séparation. Les 
deux enfants présents lors de la toute dernière séance y emporteront d'ailleurs le livre de La Tempête pour les derniers adieux.

Vignette 4: Lorsque nous le rencontrons une année après la fin des séances, l'un des pères nous rapporte que l'histoire de La Tempête fait désormais partie du quotidien dans sa maisonnée. Ce père et sa fille se la racontent de mémoire, cette dernière le réprimandant s'il oublie de mentionner l'un des objets ramenés dans le lit-radeau par le père-souris de l'histoire.

Il y a là matière à réfléchir sur ce qui fait l'isolement de certaines familles réfugiées en milieu urbain, et qui font parfois tout pour éviter de quitter leur maison. Ce ne serait pas forcément l'insécurité objective dans le pays d'accueil qui produit ce confinement. Explorer l'implicite des faits un réfugié a quitté sa maison et s'en cherche une nouvellepermet de saisir ce qui est en jeu, en deçà et au-delà du bien matériel et de sa perte. On ouvre alors la porte à ce que, même dans le refuge temporaire, quelque chose se restaure des fonctions de contenant et de protection attachées à la maison. La relation au père peut jouer un rôle de refuge et permettre aux enfants de se sentir des passagers en sécurité.

\section{La fonction de père}

Vignette 6: «Le bien être du père est intimement lié à celui de l'enfant", "ma fille est capable de me faire sortir de mon humeur maussade ... vous prenez en votre enfant ce qu'il y a de fort ... ils ont la capacité de vous faire oublier. » Ce qui se dit d'emblée du côté des pères, c'est l'intensitédu lien qui les unit à l'enfant. Puis les mots glissent vers le champ des représentations, celles que les pères se font d'eux-mêmes en relation avec leurs enfants: «Je suis incapable de faire respecter les droits de mon enfant", "Nous sommes faibles mais nos enfants ne nous voient pas comme cela»; et aussi en relation avec leur propre géniteur: "Mon père était violent et je n'ai pas voulu faire comme lui ", "Nous ne pouvions pas nous opposer à nos pères", "Je me souviens que j'avais peur de mon père».

Ces pères regardent la paternité sous deux angles, celui du présent et celui de l'héritage. Le présent dessine une hiérarchie des pouvoirs inversée par rapport à celle qui serait attendue: le pouvoir exercé est celui de l'enfant sur le père. Quant à l'héritage, il ne semble pas être une ressource.

Vignette 7: Le présent se raconte au travers des questions que les pères formulent séance après séance: "Comment imposer notre point de vue à cette enfant qui s'y oppose? Comment faire pour que notre fille accepte de se séparer de nous, lorsqu'il s'agit d'aller se coucher ou de se rendre à l'école?" Le présent se donne aussi à voir en séance sous les traits de l'interaction conflictuelle opposant un père et sa fille: un père malmené et tyrannisé sous nos yeux par sa fille pour qu'il accepte, avec elle et pour elle, d'enfreindre les règles (sortir en cours de séance, emmener un jouet chez elle). Elle parviendra à ses fins une fois en mettant dans la main de son père un petit morceau de pâte à modeler qu'il glissera dans sa poche de guerre lasse. Un geste dérisoire et désespéré, passé inaperçu du reste du groupe et que nous choisirons de ne pas mettre au jour; le geste d'un père qui se définit lui-même comme "expert en sa fille» mais qui précise aussi «qu’ils grandissent ensemble».

Une tension du quotidien qui s'invite dans le groupe en quête de soutien et qu'un des pères résume avec humour: "Nous nous découvrons pères à temps plein». S'occuper des enfants de telle manière est une activité à laquelle personne ne les a jamais formés ni destinés. Un «métier» dont les femmes ont la prérogative, en particulier lorsqu'il s'agit des jeunes enfants ou des petites filles, sans que n'existent de modèles alternatifs socialement acceptables auxquels les pères pourraient se référer ${ }^{32}$.

L'héritage sera parcouru dans le rire grâce au Catalogue de parents pour les enfants qui veulent en changer ${ }^{33}$. Planche après planche, ce catalogue présente une panoplie de modèles parentaux sous les traits de diverses espèces animales humanisées. Quelle que soit sa culture d'origine, chacun peut se projeter dans ces portraits humoristiques pour en dégager une personnalité et une couleur émotionnelle qui résultent d'une lecture subjective. Les noms attribués par l'auteur à ces couples n'ont pas joué de rôle dans le jeu des identifications puisque leur sens échappait à nos clients qui ne sont pas francophones. Bien qu'il ait aussi été écrit à l'usage des enfants, les thérapeutes décident de présenter ce livre aux pères.

Vignette 8: D’abord décontenancés par notre proposition décalée, ces pères irakiens reconnaissent leur propre père, en un mélange de rire et de gêne, dans le couple des "kostodabors", où le père est représenté sous les traits d'une sorte de rhinocéros au regard inquiétant. Ils s'identifient eux-mêmes avec moins de difficulté et dans une représentation signifiante de leur questionnement: les «compliqués", le couple, qu'ils ont élu sans en comprendre le nom, est représenté par deux corps inextricablement entremêlés. Les pères expliquent que cet entremêlement illustre leur propre représentation du lien père-enfant.

S'ils s'autorisent une prise de distance par rapport à l'héritage du modèle paternel, cet héritage semble cependant les laisser seuls aux prises avec un mandat idéal qui les dégage difficilement de l'emprise mutuelle avec l'enfant. On peut se demander, en effet, si ces pères, comme ceux que M. Lamour a rencontrés en France dans son expérience à la crèche ${ }^{34}$, qui «consciemment, se différencient de leur propre père, [ne sortent pas] vulnérabilisés de cette rupture 
dans la transmission». En fin de cycle, pourtant, des indicateurs laissent à penser, qu'en rebond, ils tentent d'introduire une dimension nouvelle dans leur relation avec l'enfant.

Vignette 9: Au cours des dernières séances, nous constatons que chaque dyade peut s'absenter à l'intérieur du groupe pour un moment de jeu complice et authentique. Cette qualité de présence à l'autre se confirme lors des sessions d'évaluation dyadique une fois les séances terminées: dans chaque cas, nous observons une interaction père-enfant beaucoup plus riche et spontanée que lors de l'évaluation initiale. Le plaisir du jeu est là de part et d'autre.

Mais ce plaisir n'est pas qu'un jeu d'enfant. Selon leurs propres mots, il procède du «soulagement» ressenti par les pères qui expliquent: participer à ces séances «c'était faire quelque chose de bon pour ma fille», "pour la première fois», rajoute l'un d'eux. Il apparaît également que la limite a repris du sens, au-delà de son sens de limitation: «Ici, il $\mathrm{y}$ avait des règles et ma fille était calme» (c'est le père que sa fille menait par le bout du nez qui s'exprime ainsi) ${ }^{35}$. On peut lire ces mots paternels comme une restauration à l'œuvre. Alors que le modèle de référence se rapporte au père pourvoyeur et autoritaire, les pères ici concernés semblent émerger du processus, plus à même de jouer un rôle qu'ils étoffent et s'inventent peut-être par là même, à la source d'autres référents.

\section{La boite de Pandore des questions}

Nous l'avons montré ci-dessus, ces pères n'ont pas été en reste de questionnements. Pourtant parmi les interrogations qui émergent spontanément aucune n'aborde le passé. C'est donc nous qui, en fin de second cycle, en ouvrons la boîte avec la clé suivante: «Pouvez-vous identifier parmi les questions que pose votre enfant, celles auxquelles vous ne savez pas répondre?»

Vignette 10: Toutes ces questions ont trait à l'Irak. L'une des fillettes demande régulièrement à son père: "Quand retourneronsnous à $X$ ?». Le père nous explique qu'X est un lieu que la famille a visité lorsqu'elle a dû fuir temporairement vers le nord de l'Irak les menaces dont il était l'objet. C'est un lieu auquel la petite fille est attachée par un souvenir marquant qu'elle évoque fréquemment avec son père en lui rappelant: "C'est là-bas que la rivière a mangé ma chaussure.» Le père prend conscience en rapportant ce propos quotidien au sein du groupe que la mémoire de l'enfant s'est aussi empreinte de la menace, à sa manière. Un autre père évoque un événement récent qui s'est produit en Irak: une explosion dans une église a causé une cinquantaine de morts, faisant la une de l'actualité. Le soir même, il a voulu en savoir plus et a demandé à sa fille d'interrompre son dessin animé pour regarder les informations télévisées sur une autre chaîne. Comme il n'est pas coutumier du fait, sa fille a insisté pour savoir ce qui était si important. Le père s'est alors entendu lui dire: "Il y a eu une explosion avec beaucoup de morts à Bagdad. "Les questions de la petite n'ont alors pas manqué, auxquelles le père s'est trouvé incapable de répondre.

Si ces conversations avec les pères ont lieu en présence des fillettes sans que celles-ci ne nous portent une attention apparente (comme cela aura fréquemment été le cas), en fin de séance leur humeur tourne à la colère qui se déverse sur les pères, comme en manière de signifier leur désaccord pour ce retour vers un passé douloureux pour tous. Les séances suivantes seront perturbées de tant d'absences qu'il sera impossible de cheminer plus avant. Ces absences peuvent aussi être comprises à la lecture faite ici de la colère des enfants.

Malgré les résistances et désirs d'oublis, nous ne doutons pas que le dispositif père-enfant soit un lieu de choix pour accompagner une sortie négociée du silence qui semble inévitable. Nous observons que ces expériences de violence et de rupture (du traumatisme à l'exil) ne se laissent pas empaqueter dans les greniers de la mémoire ou abandonner comme un jouet au rebut. Les pères que nous avons rencontrés l'ont compris et sont favorables à une levée du silence qui ne les laisse plus seuls en charge. J. le confirme lorsque nous le rencontrons avant la rédaction de cet article. Il rapporte les questions de sa fille après la fin du groupe: «Pourquoi ces marques sur ton corps? Où est ton magasin en Irak? Pourquoi es-tu venu en Jordanie?» Autant d'indicateurs que le roman du voyage d'affaires a fait long feu. La construction du sens pour l'enfant demeure une fonction paternelle essentielle à soutenir.

Comme il ne nous restait qu'à inventer, il nous reste désormais à continuer. Des questions essentielles sont restées en suspens qui pourraient se formuler en une seule: «La guerre d'Irak peut-elle être expliquée à mon enfant?» Nous réfléchissons aux modalités qui pourraient nous permettre de lui apporter une ou des réponses. Elles se devront d'être créatives et impliqueront la participation active, il va sans dire, de pères et d'enfants irakiens vivant dans le refuge jordanien.

\section{Conclusion}

À l'étape où nous nous trouvons, nous pouvons faire le bilan suivant: grâce à ce dispositif, une intelligence de la situation du père et de l'enfant a pu être mobilisée. Pères et enfants y ont trouvé, chacun pour leur compte, la preuve de ce que l'un était prêt à faire avec et pour l'autre, tout comme la mesure de ce qu'il ne pouvait encore accomplir. Mais quelque chose d'un processus de pensée a été remis en route à travers lequel les pères assument un rôle même s'ils cherchent encore à le définir. En ce sens, la proposition 
que le CVT leur a faite a répondu, à sa manière, au constat préliminaire d'une paternité mise en défaut.

Nous avons eu la preuve qu'il est possible d'engager des pères (qui ont pourtant été mis à rude épreuve) dans la quête de leur fonction. Ils nous ont surpris, à bien des égards, par leur ouverture et leur désir de transformation. Ils sont venus puiser en eux-mêmes une qualité qu'ils croyaient perdue ou qu'ils ne soupçonnaient pas. Pourquoi d'autres pères réfugiés ne seraient-ils pas prêts à prendre un chemin similaire, en Jordanie et ailleurs?

L'intervention aura montré que le refuge peut être un temps plein mis au profit d'une reconstruction personnelle et familiale et d'une reconstruction seconde, celle du sens, au cours d'un processus qui ne pouvait se faire avant (dans l'orbe de la violence) et qui ne sera pas la priorité de l'après (dans un pays tiers ou de retour en Irak). La reconstruction $\mathrm{du}$ sens après la violence pouvant prendre bien d'autres chemins n'est surtout pas l'apanage des psychologues et demeure une pierre angulaire dans le rétablissement des droits humains et de la capacité à vivre ensemble.

Nous comprenons que ce travail peut et sûrement doit se faire selon des modalités transgénérationnelles au sein desquelles les pères, épine dorsale de bien des fonctionnements sociaux de par le monde et dans cette région du MoyenOrient en particulier, doivent pouvoir jouer leur rôle.

\section{Notes}

1. Voir les recherches menées par Elisabeth Fivaz-Depeursinge dans son Centre détudes de Lausanne, portant spécifiquement sur les interactions familiales triadiques. Se référer également aux travaux français de Martine Lamour dans une même filiation théorico-clinique, en particulier: Martine Lamour, «Co-construire la paternalité: une expérience de recherche-action-formation à la crèche», in Jacques André et Catherine Chabert, Loubli du père, (Paris: Presses Universitaires de France, 2004).

2. Michel De Clerq et François Lebigot, Les traumatismes psychiques (Paris: Masson, 2001), 110-114.

3. Les souffrances psychiques liées à l'exil sont identifiées et prises en charge depuis plusieurs décennies par nombre de cliniciens. Voir les travaux et propositions thérapeutiques développées en France sous l'impulsion de Marie Rose Moro et de Cécile Rousseau au Canada, qui ont largement publié sur ces questions. Pour un regard critique sur ces dispositifs «pour migrants» voir les travaux d'Olivier Douville qui diverge des courants dits transculturels et propose d'autres réponses de soin.

4. Thierry Baubet, Christian Lachal, Lisa Ouss-Ryngaert, Marie Rose Moro, Bébés et traumas (Paris: La Pensée Sauvage Editions, 2006).

5. Il n'est qu'une expérience canadienne dont nous ayons connaissance développée autour de la problématique de la paternité dans le cadre d'une vaste initiative sociale pour la ville de Montréal: Martine Barrette, Rapport intérimaire d'activités du projet «Grandir sainement avec un père détenu» (Montréal: Maison Radisson Inc., 2003).

6. Ibid. supra, note $n^{\circ} 2,15-16$

7. Idem

8. Une réaction psychologique nécessitant un soin appartiendra classiquement à l'un ou plusieurs des trois grands ensembles de troubles suivants: troubles anxieux, troubles dépressifs et troubles post-traumatiques. Ces derniers sont les plus étudiés: pour une population donnée, un taux de prévalence des PTSD (Post-Traumatic Stress Disorder) est évalué. Nous retenons les données suivantes de la revue complète de la littérature épidémiologique réalisée par $\mathrm{A}$. Jolly: «Parmi des enfants irakiens âgés de 4 à 8 ans, réfugiés en Suède depuis un an, 21,4 pour cent souffrent d'un PTSD, tandis que 30,9 pour cent présentent un PTSD incomplet. (Almqvist et al.,1997). [...] Sur des victimes, kurdes pour la plupart, d'actes de torture, la prévalence du PTSD est estimée à 51,7 pour cent (Van Velsen et al., 1996).» Pour l’ensemble des données, se référer à: A. Jolly, «Epidémiologie des PTSD», Journal International de Victimologie 2.1 (2003), accessible en ligne http://www.jidv.com/njidv/ index.php/jidv05/111-jidv05/275-epidemiologie-des-ptsd.

9. Le terme client s'est imposé aux Etats-Unis pour désigner une personne bénéficiaire de soins. Lorganisation du CVT l'a adopté pour les bénéficiaires de ses projets de par le monde. C’est pour cette raison que le terme a été conservé dans cet article. Dans un contexte francophone, nous aurions privilégié le terme patient couramment en vigueur. Précisons que les clients sont soignés gratuitement dans les centres du CVT.

10. Le centre principal est situé à Amman dans le quartier populaire de Hashemi Shamali; un centre plus modeste a reçu des clients jusqu'à récemment à Irbid; ce dernier a fermé ses portes dans le cadre d'une nouvelle stratégie d'intervention en clinique mobile.

11. Nous ne disposons pas de données chiffrées évaluant la fréquentation masculine des consultations psychologiques au Moyen Orient. Dans les pays occidentaux, les hommes restent sous représentés parmi les consultants, alors que les représentations communes associées aux soins psychiques y ont peu à peu évoluées vers moins de stigmatisation (ce qui est encore loin dêtre le cas dans le Moyen-Orient où le majnun - fou en arabe - rôde encore comme un spectre). En outre, le modèle identificatoire le plus accessible pour un homme arabe, celui du dominant/fort, laisse facilement entrevoir les obstacles qui se présentent lorsqu'il s'agit pour lui de souvrir à un psychologue au sujet de ses faiblesses.

12. Lorganisation du CVT se réfère à la torture en adoptant la définition de la Convention des Nations Unies contre la torture et autres peines ou traitements cruels, inhumains ou dégradants, de 1984: Y a-t-il une référence à un texte ou législation plus précise? 
13. Nous nous appuyons sur les informations disponibles à ce jour dans la base de données du CVT. Elles concernent un échantillon représentatif de 315 clients (âgés de 18 à 75 ans). Lors de l'entretien initial, des informations socio-économiques et historiques ont systématiquement été collectées pour les 1200 clients traités, mais, au jour de cette rédaction, elles n'ont pu toutes être informatiquement traitées.

14. 8 pour cent seulement de nos clients nous sont référés par des organisations partenaires. La principale source de référence demeure notre personnel de terrain (58 pour cent des clients); viennent ensuite nos propres clients (18 pour cent) et leur famille ou amis ( 9 pour cent); enfin 7 pour cent de bénéficiaires potentiels se présentent d'eux-mêmes.

15. Il est, à notre connaissance, peu d'études qui se soient attachées à décrire spécifiquement le rôle des pères dans la société irakienne. On y trouve quelques allusions dans le document d' Helen Chapin Metz, Iraq: A Country Study. (Washington: GPO for the Library of Congress, 1988), notamment dans le chapitre «Famille et Société». Nous empruntons à cet auteur le qualificatif « d'autorité absolue», numéro de page?.

16. Ce rôle recouvre le fait d'assurer la pérennité et la sécurité financière et matérielle de la famille, mais aussi de la satisfaire dans ses désirs au-delà du nécessaire. La dépense procure en outre un certain statut de par sa visibilité sociale et inscrit par la même la famille dans un réseau de socialité. Pourvoir signifie aussi, et tout autant, concrétiser son affection paternelle et manifester sa compréhension de l'enfant. S'il satisfait au présent, le père ouvre aussi les perspectives d'une réussite future pour ses enfants (scolarité, union maritale).

17. Les enfants (particulièrement les garçons) ont culturellement pour mandat de perpétuer la famille et d'assurer son lignage, de même que de prendre soin de leurs ascendants une fois devenus adultes. La taille de la fratrie force généralement le respect dû à son géniteur.

18. AVSI Foundation Jordan (2011), Hope is Gain: Aligning Humanitarian Programs to the Needs of Displaced Iraqis in Jordan as They Define Them, p. 38, disponible sur demande à l'adresse suivante: amman@avsi.org.

19. Lorsque la situation de violence rapportée se caractérise par une mise en risque majeure de l'un ou plusieurs des membres de la famille, le CVT facilite l'accès des victimes aux services de protection disponibles au niveau national.

20. La réalité des violences domestiques parmi les populations exposées à la guerre et à la violence collective a été peu étudiée. Citons néanmoins deux recherches réalisées en 2006 et 2007, respectivement en Afghanistan et au Sri Lanka, par C. Catani et collaborateurs: elles concluent à un «lien systémique» entre les effets traumatiques de la guerre et la propension des pères à user de violence à l'encontre de leurs enfants, voir Claudia Catani, Elisabeth Schauer, et Frank Neuner, "Beyond Individual War Trauma: Domestic Violence Against Children in Afghanistan and Sri Lanka”, Journal of Marital and Family
Therapy (April 2008), disponible à l'adresse suivante: http://findarticles.com/p/articles/mi_qa3658/is_200804/ ai_n25500535/?tag=mantle_skin;content

21. Le nous fera référence, dans ce texte, aux thérapeutes. Cette appellation ne reflète pas la diversité des diplômes et du niveau d'expérience des différents intervenants. Il suffit de mentionner que Muriel Génot a occupé le rôle de thérapeute principal, garante de la qualité du soin. Impliqué dès son origine (de sa conception à ses réalisations), Muath Asfoor, conseiller psychologique, a apporté une riche contribution en qualité de co-thérapeute. Pour le premier cycle, Muriel Génot était assistée d'une traductrice extérieure à l'équipe, traduisant de l'arabe vers l'anglais et inversement. Il a été préféré pour le second cycle la participation d'une jeune conseillère, Hala Hammad, ayant fraîchement rejoint léquipe à l'époque. Assurant la traduction, elle bénéficiait dans le même temps d'une expérience clinique riche d'enseignement pour elle et à laquelle elle a contribué par la finesse de ses observations. C'est à ces titres que Muath Asfoor et Hala Hammad sont mentionnés comme coauteurs de cet article.

22. Les pères de ces groupes ont grandi dans une société qui encourage l'interaction père-fils au privilège de celle entre père et fille, en particulier lorsque l'enfant atteint l'âge de socialisation. Que quatre pères sur six se soient déplacés pour un soin avec leur fille est pour le moins révélateur de leur engagement et de la nécessité qu'il ont ressentie.

23. Si l'on se réfère au modèle piagetien décrivant le processus de développement cognitif de l'enfant.

24 Chaque dyade a été évaluée individuellement avant et après le cycle de séances en groupe. Cette évaluation s’est appuyée sur une grille d’observation mesurant la qualité de l'interaction père-enfant au cours d'une activité ludique.

25. Sont à disposition: un jeu de poupées reconstituant la composition familiale, une maison et des moyens de transport, des animaux, des cubes de construction, un éventail de soldats et équipements militaires, des livres d'histoires illustrées, de la pâte à modeler et de quoi dessiner.

26. Un premier groupe pilote (premier cycle) s'est tenu pour 6 séances du 28 juin au 26 juillet 2010 (durée limitée par le calendrier du Ramadan); il a accueilli 3 couples pèreenfant. Tous les pères inclus dans ce groupe accomplissaient en parallèle un travail de thérapie individuelle qui était en cours ou achevé au moment des séances de groupe. Le deuxième groupe s'est réuni pour 10 séances du 4 octobre au 22 décembre 2010, accueillant 3 autres couples (deuxième cycle). Les pères de ce deuxième groupe nont pas été suivi en thérapie individuelle soit parce qu'ils ne le nécessitaient pas, soit parce qu'ils n'y étaient pas prêts. Chaque groupe devait accueillir 4 dyades qui avaient été identifiées et évaluées comme pouvant bénéficier positivement d'un tel dispositif. À chaque cycle, une dyade a fait défaut, un taux d'abandon duquel les programmes psycho-sociaux sont coutumiers. 
27. Précisons seulement qu'il se situe en filiation avec les principes de fonctionnements de «la maison verte» pensée originellement par Françoise Dolto et déclinée sous de multiples formes de par le monde. Nous avons conçu l'espace et le temps de la séance comme «une aire potentielle d'expérience transitionnelle» à la manière dont $\mathrm{D}$. W. Winnicott l'a conceptualisée. Enfin, nous nous sommes imprégnés des réflexions de Paul Barrows lorsqu'il se penche sur les dispositifs impliquant les pères dans la psychothérapie de l'enfant.

28. La troisième dyade ne sera présente qu'à partir de la deuxième séance.

29. Le garçon nous livre la clé de cette scène: elle est associée dans son esprit à un accident dont son frère a été victime.

30. Florence Seyvos et Claude Ponti, La tempête (Paris: L'École des loisirs, 1993).

31. Idem: $31-32$

32. Ce dispositif spécifique ne nous a pas permis d’explorer l'impact identitaire de ce nouveau statut de "père à temps plein» qui ne pourrait se faire que par le biais d'entretiens individuels avec les pères. La suite de l'article rend compte néanmoins d'une réorganisation de ces pères faisant face à une réalité inédite dans leur rôle vis-à-vis de l'enfant.

33. Claude Ponti, Catalogue de parents pour les enfants qui veulent en changer (Paris: L'École des loisirs, 2008).

34. Ibid. Martine Lamour, note $\mathrm{n}^{\circ} 1,106$

35. La limite est reconnue par les psychologues dans ses effets structurant et rassurant pour l'enfant. La limite, entendue comme un interdit universel, s'applique à tous (adultes comme enfants) et balise le possible et l'impossible.
Souvent, ce sont les pères qui sont mis en charge de relayer cet impératif sociétal au sein de la famille. C'est une tâche difficile pour tout parent, parfois irréalisable lorsque la société ne les étaye plus (dans une société en proie à la violence et à la guerre, l'impossible est parfois devenu la règle) ou lorsque les contraintes les cernent de toutes parts (comment dire non à cet enfant qui a déjà tant à souffrir?). En s'identifiant aux thérapeutes, les pères de nos groupes ont pu (re)faire l'expérience qu'un interdit, lorsqu'il est posé dans une relation (et non dans le rejet et la violence), est aussi un message de confiance ou de considération envoyé à l'enfant qui est alors perçu et se perçoit comme capable de composer avec sa frustration.

Muriel Génot: Psychologue clinicienne, elle occupe actuellement le poste de psychothérapeute et formatrice pour l'organisation Center for Victims of Torture (CVT) en Jordanie, muriel.genot@free.fr. Le Center for Victims of Torture (CVT) est une organisation non gouvernementale indépendante fondée en 1985 dont le siège se trouve à Minneapolis (Minnesota, États-Unis). Au niveau international, le CVT opère actuellement en République Démocratique du Congo, au Kenya et en Jordanie. Son programme jordanien est cofinancé par le Bureau pour les Populations Réfugiées et Migrants (BPRM) et le Fond des Nations Unies pour les Victimes de Torture (UNV).

Muath Asfoor et Hala Hammad: Conseillers pour l'organisation CVT en Jordanie.

(C) Muriel Génot, Muath Asfoor et Hala Hammad, 2011. Cette œuvre en libre accès fait l'objet d'une licence Creative Commons Attribution-NonCommercial 4.0 International License, laquelle autorise l'utilisation, la reproduction et la distribution de l'œuvre sur tout support à des fins non commerciales, pourvu que l'auteur ou les auteurs originaux soient mentionnés et que la publication originale dans Refuge : revue canadienne sur les réfugiés soit citée. 
(C) Muriel Génot, Muath Asfoor et Hala Hammad, 2011. Cette œuvre en libre accès fait l'objet d'une licence Creative Commons Attribution-NonCommercial 4.0 International License, laquelle autorise l'utilisation, la reproduction et la distribution de l'œuvre sur tout support à des fins non commerciales, pourvu que l'auteur ou les auteurs originaux soient mentionnés et que la publication originale dans Refuge : revue canadienne sur les réfugiés soit citée. 